\title{
Neurological Signs and Symptoms
}

National Cancer Institute

\section{Source}

National Cancer Institute. Neurological Signs and Symptoms. NCI Thesaurus. Code

C3837.

A term that refers to the clinical manifestations resulting from pathologic processes that affect the central and peripheral nervous system. 